\title{
CARTESIAN PRODUCTS OF METRIC BAIRE SPACES
}

\author{
M. R. KROM
}

\begin{abstract}
For any topological space $X$ there is an associated ultrametric space $\mathscr{U}(X)$ such that the cartesian product $\mathscr{U}(X) \times Y$ with any other space $Y$ is a Baire space iff $X \times Y$ is a Baire space. Assuming the continuum hypothesis, there exists an indeterminate infinite two person game such that a cartesian product of the game with itself is determinate.
\end{abstract}

The widespread use of the Baire category theorem, which states that a complete metric space is a Baire space [13, p. 180], creates interest in a more complete identification of the Baire spaces. In [8] an example is given, using the continuum hypothesis, of a topological space $Z$ which is a Baire space and such that the cartesian product $Z \times Z$ is not a Baire space. Here we use that example to answer, negatively; the open question of whether products of metric Baire spaces are Baire [1, p. 120], [8, p. 159], and [11, p. 292]. We show how to obtain from any Baire space $X$ an associated metric Baire space $\mathscr{U}(X)$ of a simple.form (a space of countable sequences with the Baire metric [12, p. 124]) such that $X \times X$ is a Baire space iff $\mathscr{U}(X) \times \mathscr{U}(X)$ is a Baire space. The simplicity of this canonical form focuses attention on the further question of how the existence of a Baire space whose square is not Baire relates to additional axioms for set theory (axioms such as the continuum hypothesis). To sharpen that focus we also include some results that are formulated in purely game-theoretic terms.

1. Baire spaces. Let $\omega$ be the set of all nonnegative integers and for $n \in \omega$ let $\omega^{n}=\omega-\{0,1, \cdots, n\}$ and let $\omega_{+}^{n}=\omega^{n} \cup\{\omega\}$. For any sets $S, T$ and for $n \in \omega^{0}$ let ${ }^{S} T$ be the set of all functions from $S$ into $T$ and let ${ }^{n} T$ be the set of all functions from $\{0, \cdots, n-1\}$ into $T$. For any sequence $\alpha$ and integer $m$ in the domain of $\alpha$ we sometimes write $\alpha_{m}$ for $\alpha(m)$ and for a finite sequence $\beta \in{ }^{n} S$, for some $n \in \omega^{0}$ and set $S$, we also

Presented to the Society April 14, 1973; received by the editors March 16, 1973.

AMS (MOS) subject classifications (1970). Primary 54B10, 54C50, 54E35; Secondary 04A25, 04A30, 26A21, 90D05.

Key words and phrases. Baire space, Baire category theorem, topological product, axiom of choice, continuum hypothesis, infinite game, determinate game, metric space, ultrametric space.

(c) American Mathematical Society 1974 
denote $\beta$ by $\left\langle\beta_{0}, \cdots, \beta_{n-1}\right\rangle$. For a set $S$ of sets and $n \in \omega_{+}^{0}$ let $\downarrow^{n} S=\left\{\sigma \in{ }^{n} S \mid \sigma(h) \subseteq \sigma(h-1)\right.$ for all $\left.0<h<n\right\}$. For any sets $X, Y, X \times Y$ is the cartesian product and if $X, Y$ are topological spaces then $X \times Y$ is the cartesian product space. For any $n \in \omega_{+}^{1}$, any topological space $X$ and base $\mathscr{B}$ for $X$, let $\mathscr{S}^{n}(X, \mathscr{B})=\left\{f \mid f\right.$ is a function from $\bigcup_{0<h<n} \downarrow^{h} \mathscr{B}$ into $\mathscr{B}$ such that for any $0<h<n$ and $\left.\sigma \in \downarrow^{h} \mathscr{B}, f(\sigma) \subseteq \sigma(h-1)\right\}$. Then $\mathscr{S}^{\omega}(X, \mathscr{B})$ is called the set of strategies associated with $X$ and $\mathscr{B}$. A strategy $f$ is said to be stable on $\sigma \in \downarrow^{h} \mathscr{B}$ in case $f(\sigma)=\sigma(h-1)$. For $f \in \mathscr{S}^{\omega}(X, \mathscr{B})$, $n \in \omega_{+}^{\mathrm{l}}, f / n$ is the unique $g \in \mathscr{S}^{n}(X, \mathscr{B})$ such that $f$ is an extension of $g$. For $n \in \omega_{+}^{1}, U \in \mathscr{B}$, and $(f, g) \in \mathscr{S}^{n}(X, \mathscr{B}) \times \mathscr{S}^{n}(X, \mathscr{B})$, the corresponding play $[U, f, g]^{n}$ is the unique element of $\downarrow^{n} \mathscr{B}$ such that

$$
\begin{aligned}
& {[U, f, g]_{0}^{n}=U,} \\
& {[U, f, g]_{h}^{n}=g\left(\left\langle[U, f, g]_{0}^{n}, \cdots,[U, f, g]_{h-1}^{n}\right\rangle\right) \text { for odd } h<n, \text { and }} \\
& {[U, f, g]_{h}^{n}=f\left(\left\langle[U, f, g]_{0}^{n}, \cdots,[U, f, g]_{h-1}^{n}\right\rangle\right) \text { for even } 0<h<n .}
\end{aligned}
$$

TheOREM 1 (OXTOBY [10]). For any topological space $X$ and base $\mathscr{B}$ for $X$ such that $\varnothing \notin \mathscr{B}, X$ is a Baire space iff for any $U \in \mathscr{B}$ and $f \in \mathscr{S}^{\omega}(X, \mathscr{B})$ there exists $g \in \mathscr{S}^{\omega}(X, \mathscr{B})$ such that $\bigcap_{n \in \omega}[U, f, g]_{n}^{\omega} \neq \varnothing$.

Proof. First observe that in Theorem 1 of [10] (cf. also Theorem 6.1 of [9]), if $A=X$ and $B=\varnothing$ then the game $\langle A, B\rangle$ is determined in favor of (B) iff $X$ is of first category in $X$. That is, (B) does not have a winning strategy iff $X$ is of second category in $X$. But $X$ is a Baire space iff each nonempty open set is of second category in $X[13, \mathrm{p} .179]$. We characterize this stronger condition by allowing player (B), instead of (A), to make the first move (in effect to choose an open subspace on which the game will be played).

We will also need the following result which is essentially the statement obtained from Theorem 1 by restricting the functions $f$ and $g$ so that they depend only on the last term of their finite sequence arguments. Here we let $\downarrow^{\mathscr{B}} \mathscr{B}=\{\sigma \in \mathscr{B} \mathscr{B} \mid \sigma(G) \subseteq G$ for all $G \in \mathscr{B}\}$.

THEOREM 2. For any topological space $X$ and base $\mathscr{B}$ for $X$ such that $\varnothing \notin \mathscr{B}, X$ is a Baire space iff for any $U \in \mathscr{B}, f \in \downarrow^{\mathscr{B}} \mathscr{B}$ there exists $g \in \downarrow^{\mathscr{B}} \mathscr{B}$ such that, for the sequence $\left\{V_{n}\right\}$ where $V_{0}=U, V_{n+1}=f\left(V_{n}\right)$ for odd $n \in(1)$ and $V_{n+1}=g\left(V_{n}\right)$ for even $n \in(1), \bigcap_{n \in \omega} V_{n} \neq \varnothing$.

Proof. We show that the proof of Theorem 1 in [10] is sound if the strategies there depend only on the preceding move. Assume $A=\bigcup_{n \in \omega} A_{n}$ is a union of nowhere dense sets $A_{n}$ and let $g_{0}$ be as indicated on p. 160 of [10]. We describe a winning strategy for (B) that depends only on the preceding move. If $G$ is a choice made by $(\mathrm{A})$ at any point in the game then let the response for (B) be $G$ in case $G \cap \bigcup_{n \in \omega} A_{n}=\varnothing$, and otherwise 
let it be the first member of $g_{0}$ that is contained in $G-A_{m}$ where $m$ is the least positive integer such that $G \cap A_{m} \neq \varnothing$. Conversely, if (B) has a winning strategy that depends only on the preceding move then it may be regarded as a strategy of the kind described in [10].

A statement slightly stronger than Theorem 2 is true; it corresponds to the fact that the strategy we have just described for player (B) is a winning strategy depending only on the preceding move even when played against an opponent who may use complete information of all preceding moves.

An important class of topological spaces consists of those for which there exists a function (strategy) $g \in \downarrow^{\mathscr{B}} \mathscr{B}$ such that for any $U \in \mathscr{B}$ and $f \in \downarrow^{\mathscr{B}} \mathscr{B}$ the sequence $\left\{V_{n}\right\}$ as indicated in Theorem 2 is such that $\bigcap_{n \in \omega} V_{n} \neq \varnothing$. These topological spaces are called $\alpha$-favorable spaces $[1$, p. 116]. By Theorem 2 above, the Baire spaces which are not $\alpha$-favorable are exactly those topological spaces for which neither player has a winning strategy in the game used to define $\alpha$-favorable [1, p. 116].

2. Ultrametric spaces. For any topological space $X$ and base $\mathscr{B}$ for $X$ such that $\varnothing \notin \mathscr{B}$, the associated countable sequence space $\mathscr{U}$ is defined by $\mathscr{U}=\left\{\sigma \in \downarrow^{\omega} \mathscr{B} \mid \bigcap_{n \in \omega} \sigma(n) \neq \varnothing\right\}$, and the topology is that given by the Baire metric, for $\sigma \neq \rho$ the distance $d(\sigma, \rho)=1 /(n+1)$ where $n$ is the least integer in $\{h \in \omega \mid \sigma(h) \neq \rho(h)\}$. An associated countable sequence space is an ultrametric space (the metric $d$ satisfies the strong triangle inequality, $d(p, r) \leqq \max \{d(p, q), d(q, r)\}[3$, p. 38, Problem 4]).

THEOREM 3. For any topological spaces $X, Y$ and any base $\mathscr{B}$ for $X$ such that $\varnothing \notin \mathscr{B}, X \times Y$ is a Baire space iff $\mathscr{U} \times Y$ is a Baire space where $\mathscr{U}$ is the countable sequence space associated with $X$ and $\mathscr{B}$.

Proof. Let $X, Y, \mathscr{B}$, and $\mathscr{U}$ be as indicated and let $\mathscr{C}$ be a base for $Y$ such that $\varnothing \notin \mathscr{C}$. For any $\sigma \in \downarrow^{\omega} \mathscr{B} \supseteq \mathscr{U}$ and $n \in \omega^{0}$ let $\sigma / n$ be the element of $\downarrow^{n} \mathscr{B}$ such that $\sigma / n(h)=\sigma(h)$ for $0 \leqq h<n$, and let $B^{n}(\sigma)=\{\rho \in \mathscr{U} \mid \sigma / n=$ $\rho / n\}$. Let $\mathscr{B}^{*}$ be the base for $\mathscr{U}$ consisting of all sets of the form $B^{n}(\sigma)$ for $n \in \omega^{0}, \sigma \in \mathscr{U}$, let $\mathscr{D}=\left\{U_{1} \times U_{2} \mid U_{1} \in \mathscr{B}, U_{2} \in \mathscr{C}\right\}$, and let

$$
\mathscr{D}^{*}=\left\{U_{1} \times U_{2} \mid U_{1} \in \mathscr{B}^{*}, U_{2} \in \mathscr{C}\right\} \text {. }
$$

For any $n \in \omega_{+}^{0}$ and $s \in \downarrow^{n} \mathscr{D}^{*}$ let $\bar{s} \in \downarrow^{n} \mathscr{D}$ such that for $0 \leqq h<n$ if $s(h)=$ $B^{k}(\sigma) \times U$ then $\bar{s}(h)=\sigma(k-1) \times U$ and for any $V=B^{k}(\rho) \times U \in \mathscr{D}^{*}$ let $\bar{V}=\rho(k-1) \times U \in \mathscr{D}$. For any $B^{k}(\sigma) \times U_{1} \in \mathscr{D}^{*}$, an immediate successor of $B^{k}(\sigma) \times U_{1}$ is a set of the form $B^{k+1}(\rho) \times U_{2}$ such that $\rho / k=\sigma / k$ and $U_{2} \subseteq U_{1}$. For $n \in \omega_{+}^{1}$ and $g \in \mathscr{S}^{n}(X \times Y, \mathscr{D})$ let $(g)^{*}$ be the unique element of $\mathscr{S}^{n}\left(\mathscr{U} \times Y, \mathscr{D}^{*}\right)$ such that if $0<m<n$ and $\sigma \in \downarrow^{m} \mathscr{D}^{*}$ then $(g)^{*}(\sigma)$ is 
the unique immediate successor $B^{k}(\rho) \times U$ of $\sigma(m-1)$ such that $g(\bar{\sigma})=$ $\rho(k-1) \times U$. For $n \in \omega_{+}^{1}, \sigma \in \downarrow^{n} \mathscr{D}$ let $g_{\sigma} \in \mathscr{S}^{n}(X \times Y, \mathscr{D})$ such that for odd $h<n, g(\sigma / h)=\sigma(h)$ and such that otherwise $g_{\sigma}$ is stable.

First assume that $U_{0}^{*} \in \mathscr{D}^{*}$ and $f_{0}^{*} \in \mathscr{S}^{\omega}\left(\mathscr{U} \times Y, \mathscr{D}^{*}\right)$ such that for any $g^{*} \in \mathscr{S}^{\omega}\left(\mathscr{U} \times Y, \mathscr{D}^{*}\right), \bigcap_{n \in \omega}\left[U_{0}^{*}, f_{0}^{*}, g^{*}\right]_{n}^{\omega}=\varnothing$. We will define $U_{0} \in \mathscr{D}$ and $f_{0} \in \mathscr{S}^{\omega}(X \times Y, \mathscr{D})$ such that for any $g \in \mathscr{S}^{\omega}(X \times Y, \mathscr{D}), \bigcap_{n \in \omega}\left[U_{0}, f_{0}, g\right]_{n}^{\omega}=$ $\varnothing$. Let $U_{0}=\sigma(k-1) \times U$ where $\sigma, k$, and $U$ are such that $U_{0}^{*}=B^{k}(\sigma) \times U$. For $n \in \omega^{1}, \quad \sigma \in \downarrow^{n} \mathscr{D}$, if $s \in \downarrow^{n} \mathscr{D}^{*}$ such that $s(h)=\left[U_{0}^{*}, f_{0}^{*} / n,\left(g_{\sigma}\right)^{*}\right]_{h}^{n}$ for $0 \leqq h<n$ and $\bar{s}=\sigma$ then let $f_{0}(\sigma)=f_{0}^{*}(s)$-, otherwise let $f_{0}$ be stable on $\sigma$. Suppose $(p, q) \in \bigcap_{n \in \omega}\left[U_{0}, f_{0}, \dot{g}\right]_{n}^{\omega}$ for some $\dot{g} \in \mathscr{S}^{\omega}(X \times Y, \mathscr{D})$. Let $\sigma=\left[U_{0}, f_{0}, \dot{g}\right]^{\omega} \in \downarrow^{\omega} \mathscr{D}$ and $s=\left[U_{0}^{*}, f_{0}^{*},\left(g_{\sigma}\right)^{*}\right]^{\omega} \in \downarrow^{\omega} \mathscr{D}^{*}$. Then $\bar{s}=\sigma$ and if $s(n)=U_{n} \times V_{n}$ for $n \in \omega$ then $q \in \bigcap_{n \in \omega} V_{n}$. Also let $\delta$ be the unique element of $\downarrow^{\omega} \mathscr{B}$ such that for $n \in \omega, \delta(n)=\rho(n)$ for any $\rho \in \downarrow^{\omega} \mathscr{B}$ such that $s(2 n+1)=B^{k}(\rho) \times U$. By the definition of $\left(g_{\sigma}\right)^{*}$, if $s(2 n+1)=B^{k}(\rho) \times U$ then $k>n$. Then $p \in \bigcap_{n \in \omega} \delta(n)$ so $\delta \in \mathscr{U}$ and $(\delta, q) \in \bigcap_{n \in \omega}\left[U_{0}^{*}, f_{0}^{*},\left(g_{\sigma}\right)^{*}\right]_{n}^{\omega}$ $\neq \varnothing$, a contradiction.

Next assume that $U_{0} \in \mathscr{D}$ and $f_{0} \in \mathscr{S}^{\omega}(X \times Y, \mathscr{D})$ such that for any $g \in \mathscr{S}^{\omega}(X \times Y, \mathscr{D}), \bigcap_{n \in \omega}\left[U_{0}, f_{0}, g\right]_{n}^{\omega}=\varnothing$. We will define $U_{0}^{*} \in \mathscr{D}_{0}^{*}$ and $f_{0}^{*} \in \mathscr{S}^{\omega}\left(\mathscr{U} \times Y, \mathscr{D}^{*}\right)$ such that for any $g^{*} \in \mathscr{S}^{\omega}\left(\mathscr{U} \times Y, \mathscr{D}^{*}\right)$,

$$
\bigcap_{n \in \omega}\left[U_{0}^{*}, f_{0}^{*}, g^{*}\right]_{n}^{\omega}=\varnothing .
$$

Let $U_{0}^{*}=B^{1}(\sigma) \times U_{2}$ where $\sigma \in \downarrow^{\omega} \mathscr{B}$ such that $U_{0}=\sigma(0) \times U_{2}$. For $n \in \omega^{0}$, $s \in \downarrow^{n} \mathscr{D}^{*}$ such that $s(n-1)=B^{k}(\sigma) \times U_{3}$ for some $k \in \omega^{0}, \sigma \in \downarrow^{\omega} \mathscr{B}$, we let $f^{*}(s)=B^{k+1}(\rho) \times U_{4}$ where $\rho / k=\sigma / k$ and $\rho(k) \times U_{4}=f(\bar{s})$. Suppose there is some $g_{0}^{*} \in \mathscr{S}^{\omega}\left(\mathscr{U} \times Y, \mathscr{D}^{*}\right)$ such that $(\sigma, q) \in \bigcap_{n \in \omega}\left[U_{0}^{*}, f_{0}^{*}, g_{0}^{*}\right]_{n}^{\omega} \neq$ $\varnothing$ for some $(\sigma, q) \in \mathscr{U} \times Y$. By the definition of $\mathscr{U}$ there is $p \in \bigcap_{n \in \omega} \sigma(n) \subseteq$ $X$. Let $s=\left[U_{0}^{*}, f_{0}^{*}, g_{0}^{*}\right]^{\omega} \in \downarrow^{\omega} \mathscr{D}^{*}$ and let $g_{0}$ be any element of $\mathscr{S}^{\omega}(X \times Y, \mathscr{D})$ such that for odd $n \in \omega$ if $s(n)=B^{k}(\lambda) \times U_{n}$ then $g(\bar{s} / n)=\lambda(k-1) \times U_{n}$. Then $\bar{s}=\left[U_{0}, f_{0}, g_{0}\right]^{\omega}$ for this $g_{0}$ and $(p, q) \in \bigcap_{n \in \omega}\left[U_{0}, f_{0}, g_{0}\right]_{n}^{\omega} \neq \varnothing$, a contradiction.

The proof is completed by applications of Theorem 1 .

Any topological space is a Baire space iff its associated countable sequence space is a Baire space (this follows from Theorem 3 using the trivial one element space for $Y$ ). Thus from Theorem 3 above and by Theorem 5 of [8] we obtain the following result.

THEOREM 4. Assuming the continuum hypothesis, there exists an ultrametric Baire space $Z$ such that the cartesian product $Z \times Z$ is not a Baire space.

3. Infinite games. For any sets $X, A$ such that $A \subseteq{ }^{\omega} X$, the infinite two-person game $G_{X}(A)$ is defined so that two players I and II successively choose elements from $X$ with player I starting [4, p. 41], [5, p. 249]. A 
play is an element $x \in{ }^{\omega} X$ and I wins the play if $x \in A$, otherwise II wins. The games $G_{X}^{* *}(A)$ are defined similarly except that both I and II may choose arbitrary nonempty finite sequences from $X$. A game is determinate if either player I or player II has a winning strategy. For any nonempty sets $A, B$, and $S$ such that $A, B \subseteq{ }^{\omega} S$ let $A \dot{\times} B=\left\{\gamma \in{ }^{\omega}(S \times S) \mid\right.$ there exists $\alpha \in{ }^{\omega} A$ and $\beta \in{ }^{\omega} B$ such that for $\left.n \in \omega, \gamma(n)=(\alpha(n), \beta(n))\right\}$. For any sets $X, A$ such that $A \subseteq{ }^{\omega} X$, we will call the game $G_{X \times X}(A \dot{\times} A)$ a square of the game $G_{X}(A)$. A play $\gamma$ in $G_{X \times X}(A \dot{\times} A)$, which is a sequence of ordered pairs of elements of $X$, is a winning play for player I iff both of the component sequences from $\gamma$ are winning for $I$ in $G_{X}(A)$ while $\gamma$ is a winning play for II iff at least one of the component sequences from $\gamma$ is a winning play for II in $G_{X}(A)$. The other analogous square of a game $G_{X}(A)$ which gives this "at least one" advantage to player $\mathrm{I}$ is the associated game

$$
G_{X \times X}\left({ }^{\omega}(X \times X)-\left(\left({ }^{\omega} X-A\right) \dot{\times}\left({ }^{\omega} X-A\right)\right)\right) .
$$

For simplification we use some notation for games in terms of the set of winning plays for player II. For any sets $A, X$ such that $A \subseteq{ }^{\omega} X$ let $(A) G_{X}=G_{X}\left({ }^{\omega} X-A\right)$. Then the square of $(A) G_{X}$ which gives the "at least one" advantage to player $\mathrm{I}$ is $(A \dot{\times} A) G_{X \times X}$. We will also use $(A) G_{X}^{* *}$ for $G_{X}^{* *}\left({ }^{\omega} X-A\right)$ and refer to $(A \dot{\times} A) G_{X \times X}$ as a square of $(A) G_{X}^{* *}$.

THEOREM 5. Assuming the continuum hypothesis, there exists an indeterminate game $(A) G_{X}$ such that the square $(A \dot{\times} A) G_{X \times X}^{* *}$ is determinate.

Proof. Let $Z$ be the Baire space described in Theorem 5 of [8] for which $Z \times Z$ is not a Baire space. Let $X$ be the set of all nonempty open subsets of $Z$ and let $A=\left\{\sigma \in{ }^{\omega} X \mid\right.$ if $\sigma \in \downarrow^{\omega} X$ then $\bigcap_{n \in \omega} \sigma(n) \neq \varnothing$ and if $\sigma \notin \downarrow^{\omega} X$ then the least $n$ such that $\sigma(n+1) \nsubseteq \sigma(n)$ is odd $\}$. Player I cannot have a winning strategy in $(A) G_{X}$ or, by Theorem 2 above, $Z$ would not be a Baire space. Player II cannot have a winning strategy in $(A) G_{X}$ or $Z$ would be $\alpha$-favorable and thus $Z \times Z$ would also be a Baire space, cf. Theorems 7.12 and 7.13 , pp. 117 and 118 of [1]. Finally, by Theorem 2 above, player I must have a winning strategy in $(A \dot{x} A) G_{X \times X}$.

THEOREM 6. Assuming the axiom of choice, there does not exist a countable set $X$ and $A \subseteq{ }^{\omega} X$ such that $(A) G_{X}^{* *}$ is indeterminate and the square $(A \dot{\times} A) G_{X \times X}^{* *}$ is determinate.

Proof. Suppose that there is a countable set $X$ and $A \subseteq{ }^{\omega} X$ such that $(A) G_{X}^{* *}$ is indeterminate and the square $(A \dot{\times} A) G_{X \times X}^{* *}$ is determinate. Player I has the winning strategy in $(A \dot{\times} A) G_{X \times X}^{* *}$ because player I has 
the "at least one" advantage there, so a projection of a winning strategy for player II in $(A \dot{\times} A) G_{X \times X}^{* *}$ would be a winning strategy for player II in $(A) G_{X}^{* *}$. Let $A$ be regarded as a topological space with the Baire metric. For $\sigma \in A$ and $n \in \omega^{0}$ let $B^{n}(\sigma)=\{\rho \in A \mid \rho / n=\sigma / n\}$ and let $\mathscr{B}=\left\{B^{n}(\sigma) \mid n \in\right.$ $\left.\omega^{0}, \sigma \in A\right\}$. Then $\mathscr{B}$ is a countable base for the topology for $A$ so $\mathscr{B}$ is a locally countable pseudo base for $A$ see p. 157 of [8]. Any finite sequence in $X$ has an extension in $A$ or else player I would have a winning strategy in $(A) G_{X}^{* *}$. By Theorem 2 above, $A$ is a Baire space with a locally countable pseudo base such that $A \times A$ is not a Baire space. This contradicts Theorem 2 of [8].

The axiom of choice is used in this proof of Theorem 6 to show that $A \times A$ is not a Baire space because the proof of Theorem 2 above depends on an application of Zorn's lemma [10, p. 160]. The axiom of choice is not needed to establish the existence of an indeterminate game $(A) G_{X}^{* *}$ with $X$ uncountable [6, p. 217] and [7, p. 204]. Can the existence of an indeterminate game $(A) G_{X}^{* *}$ whose square is determinate be established without use of the continuum hypothesis? If so, then the continuum hypothesis is not needed to show that there is a Baire space whose square is not Baire (using the topology introduced in the proof of Theorem 6). Is it consistent with the axioms of set theory without the continuum hypothesis to assume that there does not exist an indeterminate game (A) $G_{X}$ whose square is determinate ? If so, then by the proof of Theorem 5 , it is consistent, without the continuum hypothesis, to assume that the square of every Baire space is Baire, $\mathrm{cf}$. the viewpoint expressed on p. 151 of [2].

\section{REFERENCES}

1. G. Choquet, Lectures on analysis. Vol. I: Integration and topological vector spaces, Benjamin, New York, 1969. MR 40 \#3252.

2. P. J. Cohen, Set theory and the continuum hypothesis, Benjamin, New York, 1966. MR 38 \#999.

3. J. Dieudonné, Foundations of modern analysis, Academic Press, New York, 1969.

4. J. E. Fenstad, Proceedings of the second Scandinavian logic symposium, Studies in Logic and the Foundations of Math., vol. 63, North-Holland, Amsterdam, 1971.

5. D. Gale and F. M. Stewart, Infinite games with perfect information, Contributions to the Theory of Games, vol. II, Ann. of Math. Studies, no. 28, Princeton Univ. Press, Princeton, N.J., 1953, pp. 245-266. MR 14, 999.

6. J. Mycielski, On the axiom of determinateness, Fund. Math. 53 (1963/64), 205224. MR 28 \#4991.

7. - , On the axiom of determinateness. II, Fund. Math. 59 (1966), 203-212. MR 35 \#1489.

8. J. C. Oxtoby, Cartesian products of Baire spaces, Fund. Math. $49(1960 / 61)$, 157-166. MR 25 \#4055, Errata MR 26, p. 1543.

9. —_, Measure and category, Springer-Verlag, New York, 1971. 
10. J. C. Oxtoby, The Banach-Mazur game and Banach category theorem, Contributions to the Theory of Games, vol. III, Ann. of Math. Studies, no. 39, Princeton Univ. Press, Princeton, N.J., 1957, pp. 159-163. MR 20 \#264.

11. R. Sikorski, On the Cartesian product of metric spaces, Fund. Math. 34 (1947), 288-292. MR 10, 22.

12. L. A. Steen and J. A. Seebach, Jr., Counterexamples in topology, Holt, Rinehart and Winston, New York, 1970. MR 42 \#1040.

13. A. Wilansky, Topology for analysis, Ginn, Waltham, Mass., 1970.

Department of Mathematics, University of California, Davis, California 95616 